\title{
El desarrollo productivo de las Pymes en la post pandemia
}

Productive development of SMEs in the post-pandemic scenario

Fernando Ruiz ${ }^{1}$

\section{Resumen}

La pandemia causada por la COVID-19 ha impactado en la economía global y, en especial, en América Latina y el Caribe de manera significativa. Ante la magnitud de las consecuencias económicas y sociales que se anticipan, es necesario pensar en diagnósticos y propuestas de intervención para el escenario de recuperación en la post pandemia. Las pymes son las principales afectadas tanto en términos de empleo como en caída de ventas. En este artículo se analiza el contexto a nivel global y regional, se realiza un diagnóstico del problema que enfrentan las pymes y se proponen recomendaciones de política de una manera integral pensando en el corto, mediano y largo plazo.

Palabras clave: post pandemia, desarrollo productivo, pymes, estrategia, digitalización.

\section{Abstract}

The pandemic caused by COVID-19 has had a major impact on global economic activity especially, in Latin America and the Caribbean. Given the extent of the economic and social consequences that are anticipated, it is necessary to think about diagnoses and policy proposals for the recovery scenario in the post-pandemic world. Small and Medium-sized enterprises (SMEs) have been affected significantly, both in terms of employment and sales decline. In this paper a global and regional analysis is introduced, a diagnosis of the problem faced by SMEs is made and policy recommendations are given in a comprehensive way, thinking both in the short and long term.

Keywords: post-pandemic, productive development, SME, strategy, digitization.

Recibido: 31 de marzo de 2021 Aceptado: 28 de junio de 2021 Publicado: 8 de julio de 2021

${ }^{1}$ Licenciado en Relaciones Internacionales. Investigador independiente. Integrante del Centro de Estudios Prospectivos y del Centro de Estudios de la Circulación del Conocimiento Científico de la Universidad Nacional de Cuyo (UNCuyo) Mendoza, Argentina. Correo electrónico: ferruiz1988@gmail.com ID https://orcid.org/0000-0001-6819-0515 


\section{Introducción}

La pandemia de la Covid-19 ha tenido un impacto significativo sobre las pymes a nivel global, y en especial en los países de América Latina y el Caribe. Ante la magnitud de las consecuencias económicas y sociales, es clave desde la academia contribuir con diagnósticos y propuestas específicas de intervención que permitan llevar adelante políticas públicas enfocadas en el desarrollo productivo de un modo sostenible en América Latina y el Caribe. Este artículo se propone pensar en el escenario de la post pandemia, brindar recomendaciones para salir adelante y lograr una recuperación sostenible. Más allá de considerar opciones de política en el corto plazo, se propone explorar intervenciones que apunten al mediano y largo plazo para establecer un horizonte de desarrollo sostenible.

Los objetivos de este artículo son los siguientes:

- Contribuir al diagnóstico y comprensión del problema que enfrentan las pymes de un modo integral, para poder desarrollar estrategias y propuestas de intervención coordinadas entre los diferentes actores implicados en el asunto, y alineadas entre sí, tomando en consideración diferentes horizontes de tiempo (corto, mediano y largo plazo).

- Facilitar la articulación de iniciativas entre universidades, gobiernos y empresas para el desarrollo de capacidades locales de adaptación y resiliencia de las pymes al nuevo escenario derivado de la pandemia.

- Proveer un insumo estratégico sobre las problemáticas en la post pandemia, para las actividades de planificación estratégica y desarrollo.

- Contribuir al debate de ideas sobre la recuperación de las economías de la región

El artículo está organizado en tres partes. En primer lugar, se hace una introducción al contexto en dos distintos niveles de análisis. Por un lado, se hace una breve consideración del contexto global para luego enfocar en el contexto regional de América Latina y el Caribe. En segundo lugar, se realiza un diagnóstico del problema que enfrentan las pymes en la región especificada, luego se hace una descomposición en múltiples dimensiones de análisis $y$, finalmente, se definen propuestas de intervención en políticas públicas. Por último, se incluyen reflexiones y conclusiones generales sobre el tema objeto de estudio.

\section{Contexto}

\subsection{Contexto global}

La pandemia del Covid-19 ha causado un impacto significativo en la economía mundial. Aún no se puede anticipar con precisión la magnitud y el sentido de las trasformaciones económicas en curso a nivel global, pero todos los indicios apuntan a que las consecuencias sociales y económicas serán particularmente graves, sobre 
todo teniendo en cuenta la especial situación de los países en desarrollo. El freno causado por el cierre de actividades económicas, la interrupción en los intercambios mundiales, el transporte internacional y el turismo han causado un deterioro significativo de la economía mundial. Se está transitando una nueva época histórica donde el modelo de globalización, tal cual se lo conocía previamente está experimentando cambios radicales, un proceso que mostraba indicios de agotamiento ya antes de la pandemia a causa de conflictos comerciales y tecnológicos entre Estados Unidos y China. Sumado a esto, las cadenas globales de valor están en crisis y muchas empresas están replanteando sus estrategias de abastecimiento ante los crecientes riesgos de falta de insumos.

Este fenómeno de paralización de la actividad económica se produce en simultáneo a un proceso de cambio tecnológico hacia una economía digital basada en el conocimiento como principal activo, donde la tecnología tiene un rol fundamental en los modelos de negocios de las empresas. Cabe mencionar que la pandemia ha actuado como un catalizador y un acelerador de procesos de cambio previos. Fenómenos anteriores como la digitalización, el teletrabajo y el comercio electrónico se han acelerado y consolidado, por lo que es fundamental desarrollar la capacidad de resiliencia y adaptación de las pymes ante las nuevas circunstancias. Además, a raíz de los confinamientos, se han producido significativos cambios en los comportamientos y hábitos de la población, que han tenido su correlato en la desaparición de antiguos modelos de negocios y la emergencia de otros nuevos.

Como consecuencia de este nuevo contexto, según un reciente informe las pequeñas y medianas empresas son las más afectadas por el impacto del confinamiento (INTERNATIONAL TRADE CENTER, 2020). Se estima que un 20\% de las pymes en el mundo corre el riesgo de cerrar de manera permanente. El estudio además indica que un 55\% de las pymes consultadas manifestaron estar gravemente afectadas por la crisis sanitaria y económica. Además, el aislamiento ha provocado caídas de ingresos para diversos sectores económicos. Esta situación puede tener implicancias severas en el empleo global, ya que las pymes son una de las mayores fuentes de empleo. La quiebra de 1 de cada 5 pymes globales puede implicar la pérdida de un $15 \%$ de los empleos a nivel global según establece el mencionado informe.

En síntesis, la disrupción tecnológica, la fragilidad de la economía mundial, el cambio climático, la pandemia, la desigualdad y los conflictos sociales derivados de estos problemas forman parten del nuevo panorama global en el cual se deberán desarrollar políticas públicas. 


\subsection{Contexto regional}

En América Latina y el Caribe, la situación es aún más delicada por la elevada participación que tiene la economía informal, el limitado espacio fiscal de los gobiernos para impulsar políticas contra cíclicas, la significativa dependencia de la exportación de materias primas y la deficiente infraestructura tanto tecnológica como sanitaria para hacer frente a la pandemia. Las políticas de contención sanitaria para minimizar la propagación del virus han conducido a una paralización de la actividad económica, lo que afecta el normal funcionamiento de las economías en diferente medida según cada sector. Las empresas enfrentan significativas disminuciones en sus ingresos, dificultades para el acceso al crédito y un aumento en la insolvencia. Además, los trabajadores sufren un marcado deterioro en sus condiciones laborales, de empleo o ingresos lo cual conduce a un deterioro de la demanda agregada de los países y a aumentos en los niveles de pobreza.

Las pymes han sido las principales afectadas enfrentando numerosas dificultades para operar en este nuevo contexto de negocios causado por la pandemia. Estas amenazas se suman a los problemas estructurales previos y, al mismo tiempo, exceden estos desafíos históricos que han debido enfrentar en el pasado. En consecuencia, la pandemia ha potenciado aún más la situación de fragilidad de los sectores sociales más vulnerables. Entre las principales dificultades que han enfrentado las pymes se puede mencionar una caída sostenida en los niveles de ventas, unas mayores tasas de cierres permanentes, falta de infraestructura tecnológica, falta de capacitación en el personal, una baja productividad, dificultades en el proceso de digitalización, escasas reservas de efectivo y restricciones en el acceso a financiamiento. (CAF , 2020)

En términos generales se estimaba que antes de finales de 2020 podían cerrar 2,7 millones de empresas, equivalente al 19\% de todas las firmas de la región. En términos de empleo, esto generaría la destrucción de más de 8,5 millones de puestos de trabajo, esto equivale a un $8,1 \%$ del total del empleo formal en el sector empresarial y más de un quinto de los puestos de trabajos generados por las empresas micro. En el gráfico a continuación se pueden observar con mayor detalle estos datos, donde se muestra el porcentaje de empresas que podrían cerrar y el porcentaje de empleos que se podrían perder para América Latina y el Caribe a causa de la pandemia según el tamaño (CEPAL, 2020). 
Gráfico 1. América Latina y el Caribe (27 países): porcentaje de empresas que podrían cerrar y de empleos perdidos, por tamaño de empresa

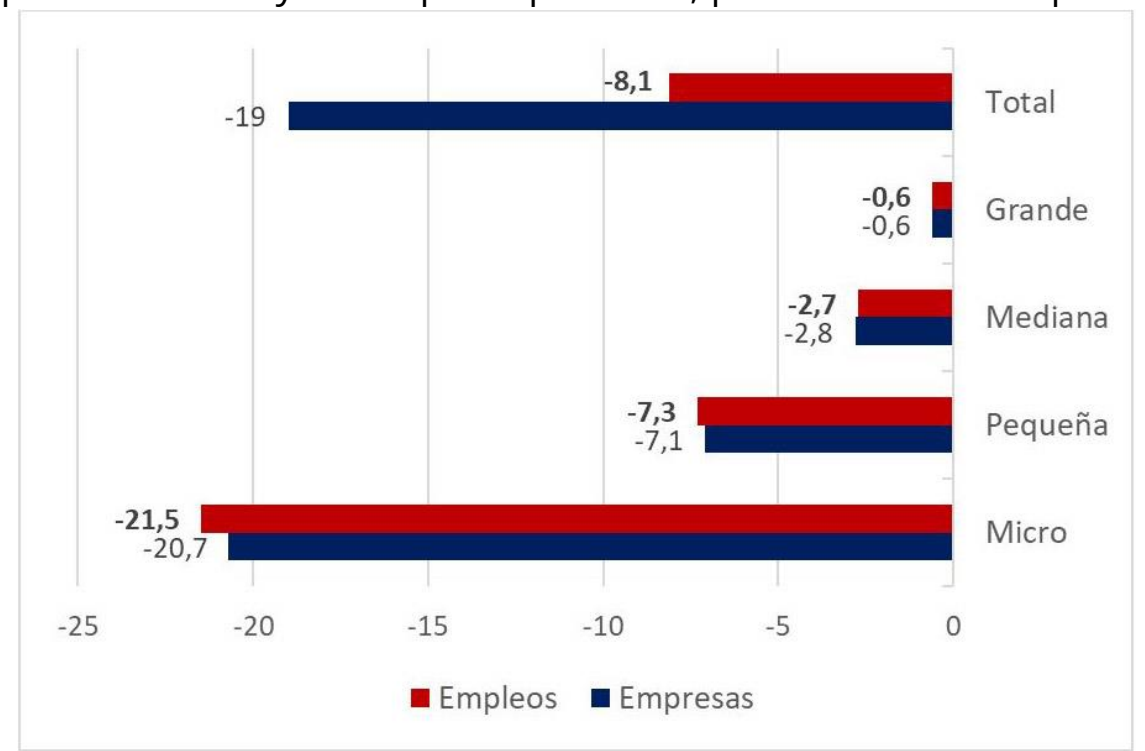

Fuente: Cepal sobre la base de datos oficiales

Sin embargo, cabe destacar que este impacto no ha sido homogéneo para todos los sectores, es decir, algunos sectores de actividad han sido más afectados que otros. El fuerte impacto registrado por las microempresas se debe, en primer lugar, a la pronunciada especialización en el sector de comercio. En 2016 aproximadamente el $42 \%$ de las microempresas de la región operaban en este rubro que ha sido uno de los más perjudicados a causa de la pandemia. Como se puede el $54 \%$ de las empresas que cerrarían pertenecen a este rubro de actividad. En segundo lugar, están las empresas dedicadas a actividades comunitarias y sociales. Finalmente, otros rubros afectados de manera significativa han sido hoteles y restaurantes, actividades inmobiliarias y la industria manufacturera. En el gráfico a continuación se muestra el número de empresas que podrían cerrar en América Latina y el Caribe según el sector de actividad al que pertenecen. 
Gráfico 2: América Latina y el Caribe (27 países): número de empresas que podrían cerrar por sector de actividad

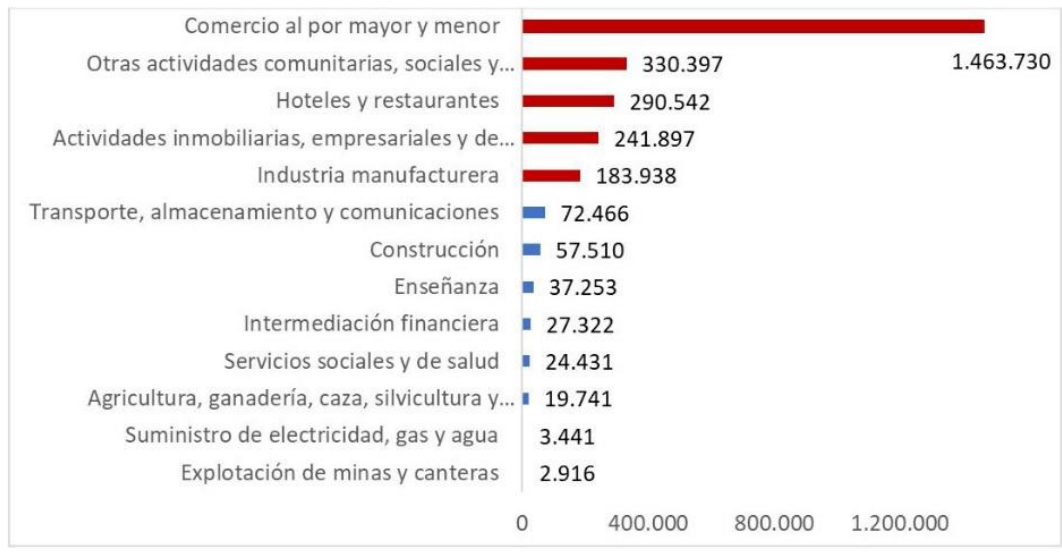

Fuente: Cepal sobre la base de datos oficiales

Sumado a lo anterior, recientemente se ha elaborado un informe para dimensionar los efectos económicos y sociales de la pandemia en los países de América Latina y el Caribe (CEPAL, 2020). De ese estudio cabe destacar tres elementos principales.

En primer lugar, la pandemia ha impactado a la región en un momento de vulnerabilidad macroeconómica. En el decenio posterior a la crisis financiera mundial (2010-2019) la tasa de crecimiento del PIB regional disminuyó del 6\% al 0,2\%; más aún el periodo 2014-2019 fue el período de menor crecimiento desde la década de 1950.

En la medida que la pandemia avanza en la región, su caracterización como crisis sanitaria, económica y social es cada vez más evidente. La dimensión y la duración de sus efectos, si bien son difíciles de cuantificar debido a la incertidumbre, comienzan a ser percibidos con claridad. Se estima que será la causa de la mayor crisis económica y social de la región registrada en décadas, con efectos muy negativos en el empleo, la reducción de la pobreza y la disminución en la desigualdad. En el mediano plazo es necesario entender los cambios estructurales en la organización de la actividad productiva que están en curso y que se intensificarán a lo largo del tiempo. Esto además tendrá impactos, en ocasiones irreversibles, en la estructura laboral, en el empleo y el bienestar de las personas. Por otra parte, la acumulación de déficit fiscales en la región ha aumentado los niveles de deuda pública de los gobiernos centrales, que en 2019 promedió el 44\% del PIB, esto representa un incremento de unos 15 puntos porcentuales respecto a su mínimo de 2011.

En segundo lugar, se estima que la pandemia llevará a la mayor contracción de la actividad económica en la historia de la región (CEPAL, 2020). La pandemia impacta a las economías de América Latina y el Caribe a través de factores tanto 
externos como internos, cuyo efecto conjunto conducirá a la peor contracción que la región ha sufrido desde que inician los registros en 1900. La economía mundial exhibe en 2020 una caída del producto bruto mayor a la observada en varias décadas. Sumado a esto el comercio mundial también se ha visto afectado significativamente.

El escenario adverso en la economía mundial impacta de manera directa en las economías, a través de múltiples canales de transmisión. El comercio exterior es afectado tanto en volumen como en precio, en especial cabe mencionar las materias primas que se exportan desde la región (petróleo, alimentos, minerales, etc.). Un factor adicional que se debe considerar es que algunos países de la región se han insertado en cadenas globales de valor, por lo que esta situación repercutirá de un modo significativo. Además, México y Centroamérica están expuestos a la contracción en las remesas que envían los trabajadores en el extranjero.

Además, las condiciones financieras a nivel mundial han sufrido un deterioro comparable al observado en la crisis de 2008. Una mayor volatilidad ha provocado salidas de capitales desde los mercados emergentes y, en consecuencia, las monedas se han depreciado respecto al dólar y los niveles de riesgo soberano han sufrido incrementos. La menor disponibilidad de flujos de financiamiento hacia economías emergentes se traduce en una ralentización de los flujos de capitales del exterior (una disminución de casi 80.000 millones de dólares respecto de 2019) y salidas netas en los flujos de cartera y otra inversión por parte de no residentes.

En tercer lugar, las proyecciones también anticipan un importante deterioro en los indicadores laborales, de pobreza y desigualdad en la región en 2020. La tasa de desempleo se ubicaría en torno al 11,5\%, un aumento de 3,4 puntos porcentuales con respecto al nivel de 2019 (8,1\%). Así, el número de desempleados de la región llegaría a 37,7 millones, lo que implicaría un aumento cercano a 11,6 millones con respecto al nivel de 2019 (26,1 millones de desempleados). La alta tasa de informalidad laboral de la región hace muy vulnerables a los trabajadores a los efectos de la pandemia y a las medidas para enfrentarla. La elevada participación de las pymes en la creación del empleo aumenta los efectos negativos, pues este sector ha sido duramente afectado por la crisis. Sumado a esto, la desigualdad de género se ha acentuado por medidas como el cierre de escuelas, el aislamiento social y el aumento del número de personas enfermas, pues ha aumentado la sobrecarga de trabajo no remunerado de las mujeres.

\section{Intervenciones de políticas}

Como se pone en evidencia en el contexto planteado, el futuro de la economía regional dependerá en gran medida de las políticas e intervenciones público-privadas que se desarrollen para contener los efectos negativos de la pandemia. El desarrollo 
productivo y la inserción global de las pymes en este contexto deberá replantearse, con distintas estrategias e intervenciones adaptadas a las nuevas dinámicas globales.

Para realizar estas intervenciones es necesario contar previamente con información estratégica actualizada al nuevo escenario global, realizar un diagnóstico de capacidades locales de las pymes, identificar y priorizar los sectores en donde hay posibilidades de desarrollo y movilizar esfuerzos para reconvertir sectores o facilitar el surgimiento de nuevos negocios en nichos con potencial. El enfoque prospectivo puede ayudar a comprender la complejidad del contexto y servir de insumo para futuras políticas sectoriales en un mundo complejo e incierto.

Cabe destacar la importancia de mejorar la competitividad y capacidades de las pymes en América Latina y el Caribe, en especial mejorando las capacidades de planificación y proponiendo estrategias de adaptación en contextos cambiantes y volátiles. Por otro lado, es fundamental nutrir la conversación estratégica de los actores implicados y ayudar a responder de manera ágil a los nuevos desafíos que presenta el mundo.

En respuesta al problema identificado anteriormente, es decir, cómo responder ante la crisis de las pymes en América Latina y el Caribe en el escenario de la post pandemia, a continuación, se realiza una descomposición del mismo en sus múltiples partes para poder comprenderlo de una manera integral y luego estar en condiciones de priorizar políticas públicas. En síntesis, se deben desarrollar estrategias y propuestas de intervención inteligentes y ágiles para afrontar las consecuencias económicas y sociales de la pandemia. Es por esta razón que es fundamental trabajar en cuatro dimensiones críticas: desarrollo productivo, infraestructura tecnológica, capital humano y macroeconomía. De igual manera, se requiere de una combinación de medidas de corto, mediano y largo plazo. 
Gráfico 3. Análisis del problema

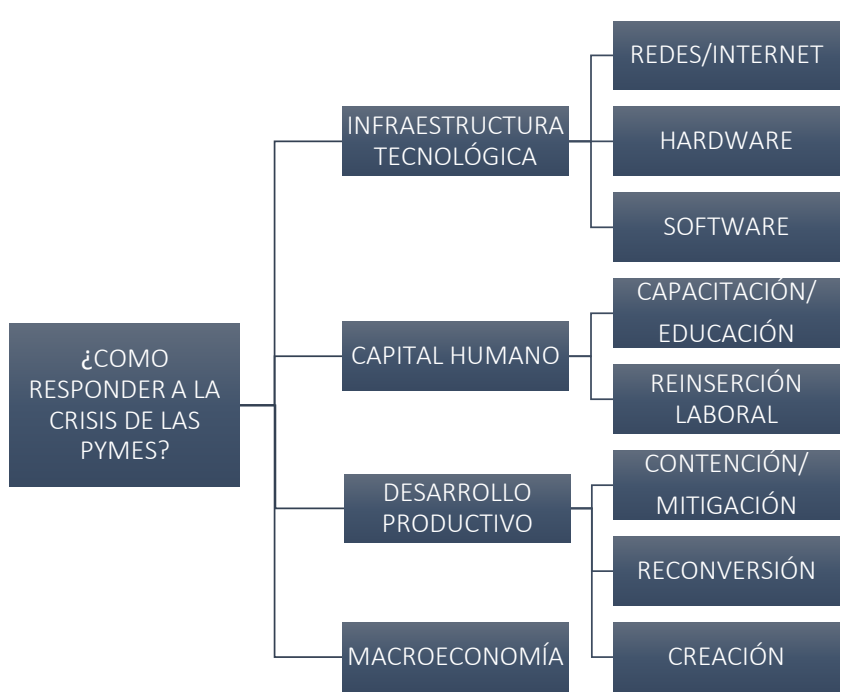

Fuente: Elaboración Propia

1.- Infraestructura tecnológica: comprender los procesos de cambios relacionados con esta dimensión implica priorizar políticas que faciliten la transformación tecnológica de las pymes, condición necesaria para operar en el nuevo contexto de negocios, es decir, se requiere generar incentivos, movilizar recursos para que las empresas se modernicen e incorporen tecnología en sus procesos. En pocas palabras, pensar en estrategias y políticas sectoriales para que las pymes puedan adaptarse al nuevo contexto global.

Esto es relevante teniendo en consideración las dinámicas globales en curso. Estas dinámicas solo beneficiarán a quienes sean capaces de innovar y adaptarse ágilmente a los cambios. En este momento se está atravesando la Cuarta Revolución Industrial, acelerada aún más por la llegada de la pandemia (SCHWAB, 2016). Esta revolución marcada por la convergencia de tecnologías digitales, físicas y biológicas transformará el mundo de una manera radical. Se pueden mencionar algunas tecnologías como la Inteligencia Artificial (IA), el Internet de las Cosas (IoT, por sus siglas en ingles), las redes sociales, la robótica, la computación cuántica, las cadenas de bloques y el cómputo en la nube. Estas tecnologías en conjunto con una mayor capacidad informática y una mayor cantidad de datos están alterando nuestras sociedades de manera profunda.

2.- Capital humano: en este punto es necesario hacer foco en aspectos relacionados con la formación técnica y profesional, en coordinación con el sector educativo en sus múltiples niveles tanto instituciones públicas como privadas. Se requiere actualizar programas de estudio, permitir a los trabajadores reinsertarse o actualizarse desde profesiones con una menor demanda en el mercado laboral a 
sectores con un mayor potencial de cara al futuro. De igual modo, resulta fundamental trabajar en brindar propuestas educativas en temas digitales en colaboración con el sector privado (marketing digital, analítica empresarial, programación, ciberseguridad, diseño web, etc.). Finalmente, se requiere promover el uso de herramientas tecnológicas para que colaboradores y empresarios puedan capacitarse, profesionalizarse y actualizarse en la gestión de sus negocios en entornos digitales.

Un tema importante relacionado al punto anterior es como facilitar la transición de empleos informales a empleos registrados. Esto es central en la región dado el alto nivel de informalidad en las economías de la región. En un reciente estudio se consultó a 800 ejecutivos de diversas industrias, los resultados de la encuesta sugieren que la crisis de la pandemia ha acelerado tendencias que estaban en desarrollo previamente y que han adquirido un nuevo ímpetu como la automatización de tareas, el trabajo remoto y la creciente demanda de trabajadores para tareas específicas que requieren un alto nivel de cualificación (McKinsey Global Institute, 2021). La pandemia ha causado una gran disrupción en los mercados laborales en el corto plazo, y puede cambiar el modo en que se trabaja en el largo plazo también. No solo la crisis actual ha lanzado a millones de personas al desempleo, sino que también el mix de empleos que surjan de esta crisis será radicalmente distinto a los empleos que se han perdido. Los sectores de menores ingresos e informales con un nivel educativo inferior serán los principales afectados, de no abordarse este problema a futuro.

3.- Desarrollo productivo: en esta dimensión es necesario desplegar acciones en tres niveles: mitigación, reconversión y creación de empresas. En el primer nivel de mitigación se trata de contener los efectos o impactos causados por la pandemia para sostener el nivel de empleo y la producción. Estas medidas ya han sido abordadas de alguna manera por los gobiernos de la región a través de distintos tipos de ayudas y mecanismos de asistencia. Los gobiernos de la región han demostrado tener la capacidad de reaccionar a los desafíos impuestos por la pandemia mediante esfuerzos para adaptar los instrumentos de apoyo y ajustar sus modalidades de gestión a las nuevas necesidades. En especial, se han desarrollado diversas políticas en cuatro áreas principales: sostener la liquidez de las empresas en el corto plazo, proteger el mercado laboral, apoyar la producción y brindar financiamiento a través de distintos programas (HEREDIA ZURITA, Andrea; DINI, Marco, 2021). Sin embargo, es necesario superar la gestión pública de un modo reactivo ante los problemas solo cuando acontecen $y$, en consecuencia, se requiere pasar a un enfoque proactivo basado en la anticipación de cara al futuro. 
El segundo nivel en el cual se puede trabajar es en el plano de la reconversión o transformación que apunta a reorientar aquellos sectores imposibilitados de continuar con sus actividades como resultado de la pandemia. En este punto incide la capacidad de los empresarios de interpretar el nuevo contexto y adaptarse de manera ágil a las nuevas circunstancias. Cabe agregar que el sector público y las universidades también tienen un rol activo que cumplir, asistiendo al sector privado ante estas reconversiones proporcionando servicios de consultoría, conocimientos y herramientas que faciliten estos procesos de reconversión.

Por último, es necesario generar políticas para el nacimiento de nuevos emprendimientos y el desarrollo de redes en el sistema de innovación. En este punto, resulta fundamental articular sistemas de innovación entre distintos actores y políticas que incentiven la actividad emprendedora.

4.- Por último, es clave pensar en los factores macro que influyen en el normal funcionamiento de las pymes. Se pueden mencionar la estructura de impuestos, financiamiento, inflación y tipo de cambio. La estabilidad macroeconómica es una condición sine qua non que permite un contexto propicio para el desarrollo de la inversión y la creación de empleos de calidad a largo plazo.

Sin un contexto de negocios que permita cierta estabilidad y brinde las condiciones necesarias para que los actores de la economía pueden proyectar a futuro el desarrollo de sus actividades, difícilmente se creará el contexto propicio para el nacimiento de nuevos negocios. Esto es evidente en la situación actual de la región, donde el cambio continuo en las reglas y la incertidumbre política perjudica el desarrollo sostenible. La evolución del pensamiento económico ha puesto de nuevo a las instituciones en el centro del debate sobre el desarrollo sostenible (PERRY, 2005). La inseguridad jurídica es una de las grandes limitantes de la inversión a largo plazo en los países de América Latina y el Caribe.

Cabe agregar otros factores relacionados, tales como la inestabilidad monetaria, el endeudamiento y las cuentas fiscales. En el fondo de estas cuestiones subyace el problema acerca del rol que debe cumplir el Estado en la sociedad y los servicios públicos que este debe brindar. En pocas palabras, es necesario un Estado ágil, eficiente y efectivo que brinde servicios públicos de calidad, sobre todo a los sectores más vulnerables de la sociedad. Para alcanzar estos objetivos es necesario previamente, plantear reformas en distintos frentes en el Estado, en especial en su estructura, capacidades, incorporación de tecnología y gestión.

\section{Conclusiones}

En la nueva normalidad se presentan tanto oportunidades como amenazas. Desde el punto de vista de las oportunidades, cuando finalice este momento bisagra 
los hábitos de consumo y comportamiento de la población habrán cambiado de manera significativa. En consecuencia, el contexto de negocios se habrá transformado radicalmente, lo cual puede ser una oportunidad para el nacimiento de nuevos emprendimientos que presenten modelos de negocios diferentes incorporando nuevas tecnologías y considerando el medio ambiente. Por lo tanto, nuevos sectores emergerán y otros enfrentarán dificultades para recuperarse dadas las nuevas condiciones de contexto.

Desde el lado de las amenazas se pueden mencionar, en primer lugar, el nuevo contexto de la globalización que emerge tras la pandemia, en especial el impacto que ha causado la pandemia en las cadenas de suministros globales (UNCTAD, 2020). Ante un escenario de mayor incertidumbre, cabe esperar que empresas y gobiernos busquen alternativas ante los riesgos crecientes, para no ver afectada su capacidad de producción y fortalecer la resiliencia frente a shocks externos. En consecuencia, esto último implica una nueva normalidad que obligará a las empresas a adaptarse ante nuevas condiciones para realizar negocios en las nuevas dinámicas globales. Cabe mencionar también otras amenazas como la desigualdad, la baja productividad, la fragilidad económica y la economía informal en el desempeño de las pymes que han sido problemas persistentes, los cuales ya estaban presentes previamente y se han profundizado con la llegada de la pandemia.

En conclusión, cabe destacar tres puntos importantes que surgen del artículo que es necesario destacar a modo de conclusión.

En primer lugar, es fundamental repensar las políticas de desarrollo productivo para el escenario de la recuperación. La aceleración del proceso de transformación digital en las empresas ha encontrado a algunos sectores mejor preparados que otros para enfrentar este cambio de época. En consecuencia, es necesario pensar en estrategias inclusivas para que los sectores más rezagados puedan incorporarse y dispongan de una mayor capacidad de adaptación. Las consecuencias económicas y sociales serán duraderas y es esencial pensar en políticas y planes de intervención para no solo mitigar y contener daños, sino también pensar en la recuperación productiva y el desarrollo en el largo plazo, de la mano de políticas sociales para integrar a los sectores más vulnerables.

En segundo lugar, es clave pensar de un modo integral analizando todas las dimensiones del problema para diseñar políticas enfocadas no solo en el corto plazo sino también en el largo plazo. De igual manera, es preciso diseñar políticas sectoriales específicas para brindar asistencia técnica y herramientas de financiamiento de acuerdo a las necesidades particulares de cada sector productivo. Finalmente, resulta fundamental pasar de la reacción a la anticipación proactiva en las políticas públicas. 
Tercero, es fundamental el proceso participativo de todos los actores implicados en el desarrollo productivo. Sin un proceso participativo de múltiples actores, es dificultoso alcanzar consensos básicos. La academia, el sector privado, y el gobierno son claves en la coordinación, la planificación e implementación de políticas públicas. En consecuencia, es clave generar espacios de participación y articulación entre estos actores.

\section{Referencias bibliográficas}

CAF . (27 de Noviembre de 2020). CAF - Banco de Desarrollo. Recuperado el 26 de Junio de 2021, de https://www.caf.com/es/conocimiento/visiones/2020/11/la-doblepandemia-de-las-pymes-latinoamericanas/

CEPAL. (2020). Dimensionar los efectos de la pandemia para pensar en la reactivación. Santiago de Chile.

CEPAL. (2020). Informe sobre el impacto económico en América Latina y el Caribe de la enfermedad por coronavirus. Santiago de Chile.

CEPAL. (20 de Diciembre de 2020). Mipymes y el COVID-19. Recuperado el 27 de Junio de 2021, de https://www.cepal.org/es/euromipyme/mipymes-covid19

HEREDIA ZURITA, Andrea; DINI, Marco. (2021). Análisis de las políticas de apoyo a las pymes para enfrenta la pandemia de COVID-19 en América Latina. Santiago: CEPAL.

INTERNATIONAL TRADE CENTER. (2020). Covid-19: The great lockdown and its impact on small business. SME competitiveness outlook. Geneva.

McKinsey Global Institute. (2021). The future of work after COVID-19. McKinsey. PERRY, G. (2005). Crecimiento en América Latina: en busca del tiempo perdido. Bogotá, Colombia.: Banco Mundial.

PRICE WATERHOUSE COOPERS. (2020). Septima encuesta a Pymes de PWC Argentina. Expectativas Mayo, 2020. Buenos Aires.

SCHWAB, K. (2016). La cuarta revolución industrial. Madrid: Debate.

UNCTAD. (2 de Septiembre de 2020). UNCTAD. Recuperado el 25 de Junio de 2021, de https://unctad.org/es/node/27709 